\title{
Selbstwechselwirkung von Gravitationsfeldern schnell bewegter Pol-Dipolquellen
}

\author{
F. BeNNEWitz und K. WestPfahl \\ Institut für theoretische Physik der Universität Freiburg (Brsg.) \\ Eingegangen am 19. April 1971
}

\begin{abstract}
We construct retarded solutions of the second order Lorentz-covariant approximation of Einstein's field equations for rapidly moving pole-dipole-like mass distributions. As the conventional Green's function method breaks down here, the integration is accomplished by a new regularization procedure based on analytic continuation of properly cut integrals. It is pointed out that there is no self-interaction for a pole-particle, in contradistinction to a spin-particle, where the self-interaction manifests itself in a backscattering of gravitational radiation (producing a wave tail).
\end{abstract}

\section{Einleitung}

Die allgemeine Relativitätstheorie führt notwendig zu einer Selbstwechselwirkung des Gravitationsfeldes, die sich in der Nichtlinearität der Einsteinschen Feldgleichungen manifestiert. Insbesondere beruht hierauf die Möglichkeit, die Bewegungsgleichungen gravitierender Teilchen aus den Feldgleichungen selbst abzuleiten ${ }^{1}$.

Ein vielversprechender Zugang zu diesem Bewegungsproblem besteht darin, die Teilchen durch enge, zeitartige Weltröhren zunächst aus dem betrachteten Feldgebiet auszuschließen und die Abweichungen von der Minkowski-Metrik außerhalb der Weltröhren als kleine Störung zu behandeln. Dieses Verfahren führt zu einer Charakterisierung der Teilchen durch die Eigenschaften der Lösungen der linearisierten Vakuum-Feldgleichungen und der an sie anschließenden Störungsrechnung, ohne auf die Konstitution der Teilchen (d. h. das Innere der Weltröhren) eingehen zu müssen. Die Extrapolation dieser Lösungen in das Innere der Weltröhren führt einerseits bei Wahrung der Euklidischen Topologie zu zeitartigen Liniensingularitäten (Materie als Feld-

${ }^{1}$ Unter Teilchen sollen dabei zunächst räumlich eng begrenzte Raum-Zeit-Bereiche mit nicht verschwindenden Gravitationsquellen (Materietensor) verstanden werden (oder auch Bereiche mit so starker Selbstwechselwirkung, daß eine Unterscheidung zwischen Feld und Quelle ihren Sinn verliert). Der folgende Abriß dient der Präzisierung unserer Auffassung des Bewegungsproblems, ohne hier auf kontroverse Standpunkte eingehen zu können. 
singularität) und läßt andererseits die Möglichkeit singularitätenfreier Teilchenmodelle mit mehrfach zusammenhängender Topologie offen (Geometrodynamik: Materie als Feldknoten).

Für quasistationäre Gravitationsfelder liefert die dann als EIHVerfahren [1] bezeichnete Methode die (konservative) nach-Newtonsche Näherung für die Bewegungsgleichungen frei gravitierender Massenpunkte. Bei den höheren (nichtkonservativen) Näherungen muß das quasistationäre Umgebungsfeld langsam bewegter Teilchen mittels einer singulären Störungsrechnung an das asymptotische Strahlungsfeld angekoppelt werden [2].

Zur Behandlung von Strahlungsproblemen dürfte es jedoch zweckmäßiger sein, ein Störungsverfahren für schnell veränderliche Felder zu verwenden, das heißt, eine Lorentz-kovariante Störungsrechnung für schnell bewegte Teilchen (auch als Entwicklung nach der Gravitationskonstanten bekannt). Dabei tritt die Wechselwirkung zwischen Feld und Teilchen dadurch zutage, daß Feld- und Bewegungsgleichungen in abwechselnden sukzessiven Schritten gelöst werden müssen.

Demgemäß hat man in nullter Näherung von einer konservativen Bewegung der Teilchen auszugehen (z. B. gleichförmig bewegte Massenpunkte oder konservative Multipolteilchen [3-5]). Der nächste Schritt liefert das Feld erster Näherung, das zu einer lichtartigen Ausstrahlung von gravitativem Feldimpuls führt. In den Bewegungsgleichungen erster Näherung [6-12] findet sich der Rückstoß dieser Impulsabstrahlung als Gravitationsstrahlungsdämpfung wieder. In zweiter Näherung [13-15] gehen nicht nur von den Teilchen selbst retardierte Gravitationswirkungen aus, sondern auch von der über den ganzen Raum ausgebreiteten Energie-Impulsverteilung des Feldes erster Näherung. Dieser Selbstwechselwirkungseffekt führt insbesondere 74 einer Selbststreuung der Gravitationsstrahlung, die sich dadurch manifestiert, daß nicht nur die retardierten, sondern auch alle vorausgegangenen Bewegungszustände der Teilchen zum Feld beitragen. Ein derartiger, über die Vorgeschichte der Teilchen summierender Feldbestandteil wird als Schweif (tail) bezeichnet: er bringt die Ausbreitung von Gravitationswirkungen (zweiter Näherung) mit beliebigen Unterlichtgeschwindigkeiten zum Ausdruck.

Eine konsistente mathematische Formulierung dieses anschaulichen Sachverhalts ist bisher allerdings dadurch erschwert worden, da 3 das Feld zweiter Näherung auf den ersten Blick überall divergiert [13-15]. Dies ist einer der Gründe dafür, daß sich die jüngste Entwicklung der Theorie der Gravitationsstrahlung [16-26] im wesentlichen auf die asymptotischen Eigenschaften der Gravitationsfelder (und ihrer Störungstheorie) konzentriert hat. Diese lassen jedoch nur indirekte Schlüsse auf die Quellen und ihre Bewegung zu. 
In der vorliegenden Arbeit soll nun gezeigt werden, daß auch bei den oben skizzierten Teilchenproblemen die Divergenzen der Störungsrechnung durch eine sorgfältigere Handhabung der Methode der Greenschen Funktion vermieden werden können (Regularisierung). Wir knüpfen dabei an eine Arbeit von Bertotti u. Plebański [13] an, in der die Feldgleichungen (zweiter Näherung) für ein System von frei gravitierenden Massenpunkten formal integriert worden sind. Da der Energie-Impulstensor des Feldes erster Näherung bilinear aus den retardierten Potentialen der Teilchen aufgebaut ist, so zerfällt das Feld zweiter Näherung in zwei Summen, von denen die eine über alle einzelnen Teilchen läuft (Einteilchenpotentiale), die andere über alle möglichen Teilchenpaare (Zweiteilchenpotentiale). Der Formalismus von Bertotti u. Plebański läßt sich jedoch nur für die Berechnung der Zweiteilchenpotentiale verwenden.

Für die Quellen der Einteilchenpotentiale (die stärkere Singularitäten aufweisen, als die Quellen der Zweiteilchenpotentiale) stellen die mit Hilfe der Greenschen Funktion $D^{\text {ret }}$ gebildeten Integrale $\int d y D^{\text {ret }}(x-y) \ldots$ selbst bei Konvergenz i.a. keine Lösung der Wellengleichung dar. Eine Lösung ergibt sich jedoch dann, wenn wir erstens den Integrationsbereich durch eine raumartige Hyperfläche in zwei Teile zerlegen, zweitens die Inhomogenität durch Einführung eines komplexen Parameters $\lambda$ geeignet abändern und schließlich drittens die Teilintegrale in der $\lambda$-Ebene getrennt analytisch fortsetzen.

Die Anwendung dieses Verfahrens auf die Einteilchenpotentiale frei gravitierender Massenpunkte liefert einfach für jedes Teilchen eine Lorentz-transformierte Schwarzschildmetrik (zweiter Näherung) und somit keinen Schweifterm. Damit verschwindet auch die über eine weitere Regularisierung zu gewinnende Selbstkraft: Für Polteilchen gibt es keine gravitative Selbstwechselwirkung (im Gegensatz zur elektrodynamischen Selbstwechselwirkung einer Punktladung [27,28]). Die durch die Zweiteilchenpotentiale gegebene Wechselwirkung der Polteilchen erscheint als ein Doppelintegral über die Vorgeschichte aller kausal nicht korrelierten (raumartigen) Teilchenpaare (Doppelschweif).

Im Gegensatz zum Polteilchen ist ein einzelnes Spinteilchen (PolDipolteilchen) ein gravitativ selbstwechselwirkendes System. Dies äußert sich einmal im Auftreten von Selbstkräften in den Bewegungsgleichungen erster Näherung [12], zum andern durch die Selbststreuung von Gravitationsstrahlung im Feld der zweiten Näherung (wie als weitere Anwendung unseres Verfahrens gezeigt werden soll). In der Tat besitzt dieses Feld einen Schweifterm, dessen physikalische Interpretation weiter oben erläutert worden ist (Selbststreuung, Wirkungsausbreitung mit Unterlichtgeschwindigkeit). 
Im einzelnen gliedert sich die Arbeit wie folgt: Nach einer Zusammenstellung der Feld- und Bewegungsgleichungen in zweiter Lorentzkovarianter Näherung $(\S 2)$ entwickeln wir unser Regularisierungsverfahren für die hier auftretenden singulären Feldquellen (§3). Die Anwendung auf Pol- und Spinteilchen erfolgt in $\S 4$.

\section{Grundlagen}

\subsection{Feld- und Bewegungsgleichungen}

Wir betrachten bezüglich der Koordinatensubstitutionen

$$
x^{\alpha} \rightarrow x^{\alpha}+\eta^{\alpha}(x)
$$

kovariante Riemannsche Maßtensoren der Form

$$
g_{\alpha \beta}(x)=\eta_{\alpha \beta}+h_{\alpha \beta}(x),
$$

wobei der Minkowskische Maßtensor

$$
\eta_{\alpha \beta}=\operatorname{diag}(1,-1,-1,-1)
$$

als invariant und die Größen $\eta^{\alpha}$ und $h_{\alpha \beta}$ (sowie ihre sämtlichen ersten und zweiten partiellen Ableitungen) als von gleicher Ordnung zu behandeln sind. Die Einstein-Gleichungen lassen sich dann formal in ein unendliches System sukzessiv zu lösender Gleichungen der allgemeinen Form $^{2}$

$$
\square \hat{h}_{\alpha}^{\beta}=-2 x \Theta_{\alpha}^{\beta}, \quad \hat{h}_{\alpha \mid \beta}^{\beta}=0
$$

aufspalten, welches mit der als Erhaltungssatz auftretenden Integrabilitätsbedingung

$$
\Theta_{\alpha \mid \beta}^{\beta}=0
$$

verknüpft ist (vgl. z. B. [29]). In der $n$-ten Ordnung ist hierbei $\hat{h}_{\alpha}^{\beta}$ ein Polynom $n$-ten Grades in $h_{\alpha}^{\beta}$ und $\Theta_{\alpha}^{\beta}$ der Energie-Impuls-Tensor des betrachteten Systems in $(n-1)$-ter Ordnung (einschließlich des Gravitationsfeldes). Wenn wir die $\hat{h}_{\alpha}^{\beta}$ als primäre Feldgrößen auffassen, dann

${ }^{2}$ Griechische Indices laufen von 0 bis 3 und unterliegen der Summenkonvention. Indexbewegungen sind ausnahmslos mit dem Minkowskischen Maßtensor durchzuführen. $\varkappa=8 \pi \gamma$ ist die Einsteinsche, $\gamma$ die Newtonsche Gravitationskonstante. Die Einheiten seien so gewählt, daß die Lichtgeschwindigkeit den Wert $c=1$ hat. Indexklammern haben folgende Bedeutung:

$$
A_{(\alpha \beta)}=A_{\alpha \beta}+A_{\beta \alpha}, \quad A_{[\alpha \beta]}=A_{\alpha \beta}-A_{\beta \alpha} .
$$

Partielle Ableitungen nach den Koordinaten des Feldpunktes $x^{\alpha}$ kennzeichnen wir durch $\partial_{\alpha} A=A_{\mid \alpha}\left(\partial_{\alpha} \partial^{\alpha}=\square\right)$. Bei Skalarprodukten lassen wir häufig die verjüngten Indices weg: $u_{\alpha} R^{\alpha}=u R, R_{\alpha} R^{\alpha}=R^{2}$ etc. 
gilt bis zu den in ihnen quadratischen Termen $\left(\hat{h}=\frac{1}{2} \hat{h}_{\alpha}^{\alpha},-2 x \hat{h} T_{\alpha}^{\beta}=\hat{h} \square \hat{h}_{\alpha}^{\beta}\right)$ :

mit

$$
\begin{aligned}
\Theta_{\alpha}^{\beta}= & (1-\hat{h}) T_{\alpha}^{\beta}+\frac{1}{4 \varkappa}\left\{\hat{h}_{\varkappa \mid \alpha}^{\lambda} \hat{h}_{\lambda}^{\chi \mid \beta}-2 \hat{h}_{\mid \alpha} \hat{h}^{\mid \beta}+\hat{h}_{(\alpha}^{(\chi \mid \lambda)} \hat{h}_{\varkappa \mid \lambda]}^{[\beta]}\right. \\
& \left.-\left.2 \hat{h}_{\varkappa}^{\lambda} \hat{h}_{\alpha}^{\beta}\right|_{\lambda} ^{\chi}+\delta_{\alpha}^{\beta}\left[\hat{h}_{\varkappa}^{\lambda \mid \mu}\left(\hat{h}_{\mu \mid \lambda}^{\chi}-\frac{1}{2} \hat{h}_{\lambda \mid \mu}^{\chi}\right)+\hat{h}_{\mid \chi} \hat{h}^{\mid x}\right]\right\}
\end{aligned}
$$

$$
h_{\alpha}^{\beta}=(1-\hat{h}) \hat{h}_{\alpha}^{\beta}+\hat{h}_{\alpha}^{\alpha} \hat{h}_{\varkappa}^{\beta}-\delta_{\alpha}^{\beta}\left(\hat{h}-\frac{1}{2} \hat{h}^{2}+\frac{1}{4} \hat{h}_{\varkappa}^{\lambda} \hat{h}_{\lambda}^{\chi}\right),
$$

wobei $T_{\alpha}^{\beta}$ der Beitrag der Materie ist (einschließlich der Wechselwirkung mit dem Gravitationsfeld). Die Gln. (2) sind in dieser Ordnung gegenüber den durch (1a) induzierten Eichtransformationen

mit

$$
\begin{aligned}
\hat{h}_{\alpha}^{\beta} \rightarrow & \hat{h}_{\alpha}^{\beta}-\left(1-\eta^{\alpha}{ }_{\mid \chi}\right) \eta_{(\alpha}{ }^{\mid \beta)}-\eta^{\alpha}{ }_{\mid \chi} \hat{h}_{\alpha}^{\beta}+\eta_{(\alpha}{ }^{\mid \chi}\left(\hat{h}_{\varkappa}^{\beta)}-\frac{1}{2} \eta^{\beta)}{ }_{\mid \chi}\right) \\
& +\delta_{\alpha}^{\beta}\left[\left(1-\frac{1}{2} \eta^{\chi}{ }_{\mid \chi}\right) \eta_{\mid \lambda}^{\lambda}-\frac{1}{2} \eta^{\alpha}{ }_{\mid \lambda} \eta_{\mid \chi}^{\lambda}\right]
\end{aligned}
$$

invariant.

$$
\square \eta^{\alpha}=\left.\hat{h}_{\varkappa}^{\lambda} \eta^{\alpha}\right|_{\lambda} ^{\varkappa}
$$

Die Bewegungsgleichungen fließen (als notwendige und hinreichende Bedingung) aus den mit den Feldgleichungen unlösbar verknüpften Erhaltungssätzen (2b). Für das Polteilchen folgt in zweiter Ordnung ${ }^{3}$

$$
T_{\alpha}^{\beta}(x)=\int_{-\infty}^{\infty} m u_{\alpha} u^{\beta} \delta(x-\xi) d s
$$

mit ( $m_{0}$ ist die Ruhmasse des freien Teilchens)

$$
\begin{gathered}
m=m_{0}\left[1-\frac{1}{2} h_{\alpha}^{\beta} u^{\alpha} u_{\beta}+\frac{3}{8}\left(h_{\alpha}^{\beta} u^{\alpha} u_{\beta}\right)^{2}\right], \\
\dot{u}^{\alpha}=\left(p_{\beta}^{\alpha}-p_{\gamma}^{\alpha} h_{\beta}^{\gamma}\right)\left(\frac{1}{2} h_{\varkappa}^{\lambda \mid \beta} u_{\lambda}-\dot{h}_{\varkappa}^{\beta}\right) u^{\alpha} .
\end{gathered}
$$

Entsprechend für das Spinteilchen [30] $\left(P_{\alpha}\right.$ und $S_{\alpha \beta}$ sind der Impulsvektor und Spintensor des Teilchens):

mit

$$
\begin{aligned}
T_{\alpha}^{\beta}(x)= & \frac{1}{2} \int_{-\infty}^{\infty}\left[u_{(\alpha} P^{\beta)}-\frac{1}{4} S_{(\alpha}^{\varkappa}\left(\delta_{\lambda}^{\beta)}-h_{\lambda}^{\beta)}\right)\left(\dot{h}_{\varkappa}^{\lambda}+h_{\sigma \mid \chi]}^{[\lambda} u^{\sigma}\right)\right. \\
& \left.+u_{(\alpha} S^{\beta) \gamma} \partial_{\gamma}\right] \delta(x-\xi) d s
\end{aligned}
$$

$$
\dot{P}_{\alpha}=F_{\alpha}, \quad u_{[\alpha} P_{\beta]}+\dot{S}_{\alpha \beta}=N_{\alpha \beta}
$$

3 Notation:

$$
d s^{2}=\eta_{\alpha \beta} d \xi^{\alpha} d \xi^{\beta}, \quad u^{\alpha}=\dot{\xi}^{\alpha}, \quad \dot{A}=d A / d s .
$$

$\xi^{\alpha}=\xi^{\alpha}(s)$ ist die zeitartige Weltlinie des Teilchens und

$$
p_{\alpha}^{\beta}=\delta_{\alpha}^{\beta}-u_{\alpha} u^{\beta}
$$

vermittelt die Projektion in den Orthogonalraum von $u^{\alpha}$ (momentanes Ruhsystem). $\delta(x)$ ist die vierdimensionale Deltafunktion $\left(d x=d x^{0} d x^{1} d x^{2} d x^{3}\right)$ :

$$
\int f(x) \delta(x-\xi) d x=f(\xi) \text {. }
$$


und

$$
\begin{aligned}
F^{\alpha}= & -\frac{1}{2}\left(\delta_{\varkappa}^{\alpha}-h_{\varkappa}^{\alpha}\right)\left(\dot{h}_{\beta}^{\chi}+h_{\lambda \mid \beta]}^{[\varkappa} u^{\lambda}\right) P^{\beta} \\
& -\frac{1}{4}\left(\dot{h}_{\varkappa}^{\alpha}+h_{\sigma \mid x]}^{[\alpha} u^{\sigma}\right)\left[\left(\dot{h}_{\lambda}^{\alpha}+h_{\tau \mid \lambda]}^{[\varkappa} u^{\tau}\right) S^{\lambda \mu} u_{\mu}\right. \\
& \left.+S^{\varkappa \lambda} h_{\beta \mid \lambda}^{\gamma} u^{\beta} u_{\gamma}\right]+\frac{1}{2}\left(\delta_{\varkappa}^{\alpha}-h_{\varkappa}^{\alpha}\right) h_{\sigma \mid \tau}^{[\varkappa \mid \lambda]} S^{\sigma \tau} u_{\lambda} \\
& +\frac{1}{4}\left[\left(h_{[\varkappa \mid \lambda]}^{\alpha}+h_{\varkappa \lambda}^{\mid \alpha}\right) \dot{h}_{\mu}^{\alpha}+\left(h_{\lambda}^{[\alpha \mid \chi]}-h_{\mid \lambda}^{\alpha \varkappa}\right) h_{[\mu \mid \chi]}^{\sigma} u_{\sigma}\right] S^{\lambda \mu} \\
& N^{\alpha \beta}=\frac{1}{2} S^{\varkappa[\alpha}\left(\delta_{\lambda}^{\beta]}-h_{\lambda}^{\beta]}\right)\left(\dot{h}_{\varkappa}^{\lambda}+h_{\sigma \mid \chi]}^{[\lambda} u^{\sigma}\right) .
\end{aligned}
$$

In erster Ordnung sind jeweils die in $h_{\alpha}^{\beta}$ quadratischen Terme zu streichen, in nullter Ordnung auch die linearen.

\subsection{Erste Näherung}

Wir beschränken uns zunächst auf das Polteilchen. Die retardierte Lösung der Feldgleichungen

$$
\square \hat{h}_{\alpha}^{\beta}(x)=-2 \varkappa m_{0} \int_{-\infty}^{\infty} u_{\alpha} u^{\beta} \delta(x-\xi) d s
$$

läßt sich mit Hilfe der retardierten Greenschen Funktion ${ }^{4}$

mit

$$
D^{\mathrm{ret}}(x)=2 \Theta(x) \delta\left(x^{2}\right)
$$

in die Form

$$
\square D^{\text {ret }}(x)=4 \pi \delta(x)
$$

$$
\hat{h}_{\alpha}^{\beta}=-4 \alpha \int_{-\infty}^{\infty} u_{\alpha} u^{\beta} D^{\text {ret }}(R) d s=-4 \alpha \varrho u_{\alpha} u^{\beta}(\text { ret })
$$

setzen $^{5}$, wobei die Nebenbedingung $\hat{h}_{\alpha \mid \beta}^{\beta}=0$ genau dann erfüllt ist, wenn die Bewegungsgleichung $(7 \mathrm{~b})$ in nullter Näherung befriedigt ist (d. h. $\dot{u}^{\alpha}=0$ ). Am Ort des Teilchens $(x=\xi)$ läßt sich (13) auf die übliche Weise regularisieren (d. h. durch die halbe Differenz zwischen retardierter und avancierter Lösung ersetzen [27]) und man erhält mit $\dot{u}^{\alpha}=0$ die Werte (vgl. [12]):

$$
\hat{h}_{\alpha}^{\beta}(\xi)=0, \quad \hat{h}_{\alpha \mid \gamma}^{\beta}(\xi)=0 .
$$

${ }^{4} \Theta(x)$ hat innerhalb und auf dem Vorkegel von $x^{2}=0$ den Wert 1 , sonst den Wert 0. $\delta\left(x^{2}\right)$ ist die eindimensionale Deltafunktion mit dem Lichtkegel $x^{2}=0$ als Träger.

${ }^{5}$ Notation:

$$
\alpha=m_{0} \gamma, \quad R^{\alpha}=x^{\alpha}-\xi^{\alpha}, \quad \hat{\varrho}=(u R)^{-1}, \quad k^{\alpha}=\varrho R^{\alpha} .
$$

Die Retardierung (ret) kommt durch $R^{2}=0, \hat{\varrho}>0$ zum Ausdruck. Die retardierte Eigenzeit $s_{\text {ret }}$ ist im folgenden stets vor Ausführung einer $\partial_{\alpha}$-Operation einzusetzen. 
Im Hinblick auf(7) hat somit das gravitative Eigenfeld in erster Näherung keinen Einfluß auf die Bewegung des Polteilchens:

$$
m=m_{0}, \quad \dot{u}^{\alpha}=0 .
$$

Handelt es sich dagegen um mehrere frei gravitierende Polteilchen so erhält man durch Summation von (13) und Einsetzen in (7) die Bewegungsgleichungen ${ }^{6}$

$$
\begin{gathered}
m=m_{0}\left[1+\sum_{\bar{\zeta}}^{\prime} \bar{\alpha} \hat{\varrho}\left(2 \bar{u}_{0}^{2}-1\right)\right](\text { ret }) \\
\dot{u}^{\alpha}=p_{\beta}^{\alpha} \sum_{\bar{\zeta}}^{\prime} \bar{\alpha}^{2} \hat{\varrho}^{2}\left[\left(4 \underset{0}{\bar{u}} \bar{k}-2 \bar{u}^{2}-1\right) \bar{u}^{\beta}+\left(1-2 \bar{u}^{2}\right) \bar{k}^{\beta}\right](\mathrm{ret}),
\end{gathered}
$$

wobei wegen (14) über alle Teilchen $\bar{\xi}$ mit Ausnahme des herausgegriffenen $\xi$ zu summieren ist. (Die Eigenzeiten der Teilchen $\bar{\xi}$ sind bezüglich $\xi$ retardiert.)

\subsection{Zweite Näherung}

Mit dem Materietensor (6), den Potentialen erster Ordnung (13), (14) und der Massengleichung erster Ordnung (16a) ergeben sich für ein System frei gravitierender Polteilchen folgende Feldgleichungen zweiter Ordnung:

$$
\begin{aligned}
\square \hat{h}_{\alpha}^{\beta}= & -16 \pi \sum_{\xi} \alpha \int_{-\infty}^{\infty}\left[1+\sum_{\bar{\xi}}^{\prime} \bar{\alpha} \hat{\varrho}\left(1+2 \bar{u}^{2}\right)\right] u_{\alpha} u^{\beta} \delta(R) d s \\
& +4 \sum_{\xi} \alpha^{2} \int_{-\infty}^{\infty} d s \hat{\varrho}^{3} D^{\mathrm{ret}}(R)\left(3 u_{\alpha} u^{\beta}-\frac{1}{2} \delta_{\alpha}^{\beta}+u_{(\alpha} k^{\beta)}-k_{\alpha} k^{\beta}\right) \\
& +16 \sum_{\xi \bar{\xi}}^{\prime} \alpha \bar{\alpha} \int_{-\infty}^{\infty} d s \int_{-\infty}^{\infty} d \bar{s} \nabla_{\alpha}^{\beta} D^{\mathrm{ret}}(R) D^{\mathrm{ret}}(\bar{R}) .
\end{aligned}
$$

Dabei haben wir in der zweiten Doppelsumme die Glieder mit $\bar{\xi}=\xi$ abgespalten und mit Hilfe von (13) unter Verwendung der Bewegungsgleichung nullter Näherung reduziert. Bei den übrigen Summanden $\bar{\xi} \neq \xi$ läßt sich aufgrund der Tatsache, daß (13) nur von der Differenz $R=x-\xi$ abhängt, der Operator ${ }^{7}$

$$
\begin{aligned}
\nabla_{\alpha}^{\beta}= & \frac{1}{8}\left(1-2 \bar{u}_{0}^{2}\right)\left(\nabla_{(\alpha} \bar{\nabla}^{\beta)}-\nabla \bar{\nabla} \delta_{\alpha}^{\beta}\right)+\bar{u} \bar{u} \nabla\left(u_{(\alpha} \bar{\nabla}^{\beta)}-\frac{1}{2} u \bar{\nabla} \delta_{\alpha}^{\beta}\right) \\
& -\frac{1}{2} \bar{u}_{(\alpha}\left(u^{\beta)} \bar{u} \bar{\nabla}-\bar{u}^{\beta)} u \bar{\nabla}\right) u \bar{\nabla}-\frac{1}{2} u_{(\alpha} \bar{u}_{0}^{\beta)} \bar{u} \nabla \bar{\nabla}
\end{aligned}
$$

herausziehen.

${ }^{6}$ Für ein zweites Teilchen $\bar{\xi}$ versehen wir die entsprechenden Größen mit einem Querstrich. Bei der Summation $\sum_{\bar{\xi}}^{\prime}$ ist der Summand $\bar{\xi}=\xi$ auszuschließen. In (16) gilt spe-
ziell:

$$
\hat{\varrho}=(\bar{u} \bar{R})^{-1}, \quad \bar{R}^{\alpha}=\xi^{\alpha-\bar{\xi}^{\alpha},} \quad \bar{k}^{\alpha}=\hat{\varrho} \bar{R}^{\alpha}, \quad \bar{u}=u \bar{u}, \quad{ }_{0}^{k}=u \bar{k} .
$$

${ }^{7}$ Notation:

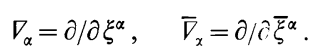



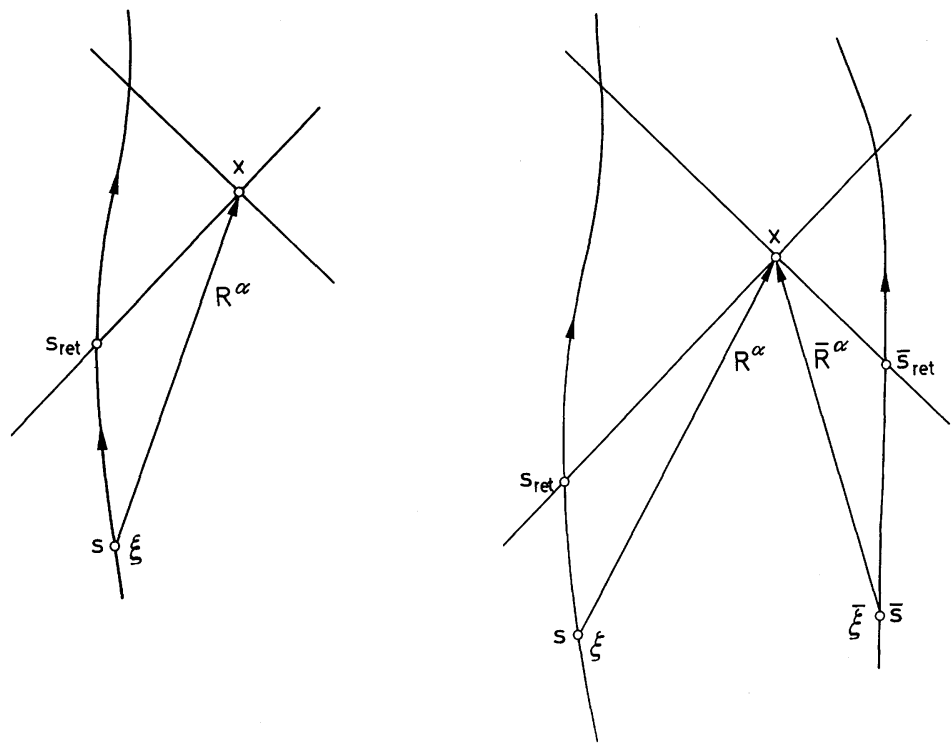

Abb. 1. Zur Berechnung der Einteilchen-Potentiale

Abb. 2. Zur Berechnung der Zweiteilchen-Potentiale

Wenn man nun zur Lösung von (17) die Greensche Funktion (11) verwendet, erhält man formal

$$
\begin{aligned}
\hat{h}_{\alpha}^{\beta}(x) \stackrel{G}{=} & -4 \sum_{\xi} \alpha \hat{\varrho}\left[1+\sum_{\bar{\xi}}^{\prime} \bar{\alpha} \hat{\underline{\varrho}}\left(1+2 \bar{u}_{0}^{2}\right) u_{\alpha} u^{\beta}\right](\text { ret }) \\
& +\pi^{-1} \sum_{\xi} \alpha^{2} \int d y D^{\mathrm{ret}}(x-y) \int d s D^{\mathrm{ret}}(y-\xi) \\
& \cdot \hat{\varrho}^{3}\left(3 u_{\alpha} u^{\beta}-\frac{1}{2} \delta_{\alpha}^{\beta}+u_{(\alpha} k^{\beta)}-k_{\alpha} k^{\beta}\right) \\
& +\pi^{-1} \sum_{\xi \xi}^{\prime} \alpha \bar{\alpha} \int d y D^{\mathrm{ret}}(x-y) \int d s \int d \bar{s} \nabla_{\alpha}^{\beta} D^{\mathrm{ret}}(y-\xi) D^{\mathrm{ret}}(y-\bar{\xi}),
\end{aligned}
$$

wobei sich die $y$-Integrationen (nach Vertauschung mit den Weltlinienintegrationen, s. Abb. 1 u. 2) ausführen lassen ${ }^{8}$ :

$$
\begin{gathered}
\frac{1}{4 \pi} \int \hat{\varrho}^{3}\left(\frac{1}{2} \delta_{\alpha}^{\beta}-3 u_{\alpha} u^{\beta}-u_{(\alpha} k^{\beta)}+k_{\alpha} k^{\beta}\right) D^{\mathrm{ret}}(x-y) D^{\mathrm{ret}}(y-\xi) d y \\
=\frac{4}{3} \Theta(R)\left[\left(\frac{1}{2} \delta_{\alpha}^{\beta}-11 u_{\alpha} u^{\beta}\right) / R^{2}\right]^{\cdot} \\
\frac{1}{4 \pi} \int D^{\mathrm{ret}}(x-y) D^{\mathrm{ret}}(y-\xi) D^{\mathrm{ret}}(y-\bar{\xi}) d y \\
=\frac{1}{2} \Theta(R) \Theta(\bar{R}) \Theta\left(-|R-\bar{R}|^{2}\right)\left[(R \bar{R})^{2}-R^{2} \bar{R}^{2}\right]^{-\frac{1}{2}} .
\end{gathered}
$$

${ }^{8}$ Siehe $\S 3$ und Anhang. Die Stufenfunktion $\Theta\left(-|R-\bar{R}|^{2}\right)$ hat den Wert 1, wenn $\xi$ und $\bar{\xi}$ raumartig oder lichtartig zueinander liegen, sonst den Wert 0 . 
Damit liefert (19) nur für die Zweiteilchenpotentiale ein konvergentes Ergebnis, während die Einteilchenpotentiale für alle Feldpunkte $x$ divergieren (da $R^{2}$ auf dem Lichtkegel verschwindet). Im folgenden ist es daher unsere Aufgabe, ein konvergentes (regularisierendes) Integrationsverfahren für die Einteilchen-Feldgleichungen zu entwickeln.

\section{Das neue Regularisierungsverfahren ${ }^{9}$}

\subsection{Grundlagen}

Zur Lösung der Wellengleichung

$$
\square \varphi(x)=g(x)
$$

für eine (weiter unten noch genauer spezifizierte) Klasse singulärer Quellverteilungen $g(x)$ schlagen wir folgenden Weg ein: Die Inhomogenität $g(x)$ wird zunächst durch Einführung eines komplexen Parameters $\lambda$ so in $g_{\hat{\lambda}}(x)$ mit $g_{1}(x)=g(x)$ abgeändert, daß das Integral

$$
4 \pi \varphi_{\lambda}(x)=\underset{\lambda}{\operatorname{anc}} f g_{\lambda}(y) D^{\mathrm{ret}}(x-y) d y
$$

(zumindest) in folgendem Sinne existiert: Wir zerschneiden den Vorkegel (Integrationsbereich) durch eine beliebige raumartige Hyperfläche in zwei Teile ${ }^{10}$ und setzen voraus, daß die beiden Teilintegrale (wenigstens) in disjunkten Gebieten der $\lambda$-Ebene konvergieren und sich von dort in ein gemeinsames Gebiet analytisch fortsetzen lassen ${ }^{11}$. Wir suchen dann eine Lösung der Ausgangsgleichung, die sich durch getrennte analytische Fortsetzung der Teilintegrale nach $\lambda \rightarrow 1$ gewinnen läßt:

$$
4 \pi \varphi(x)=\underset{\lambda \rightarrow 1}{\operatorname{anc} f} g_{\lambda}(y) D^{\mathrm{ret}}(x-y) d y .
$$

In der Tat wird sich zeigen, da $\beta$ dieses $\lambda$-Schnittverfahren eine Lösung der Einteilchen-Feldgleichungen liefert. Falls (23) bereits vor dem Zerschneiden in einem Gebiet der $\lambda$-Ebene analytisch und $\lambda=1$ ein regulärer Punkt ist, oder (23) gar unzerschnitten in einer Umgebung von $\lambda=1$ konvergiert, so führt die Regularisierung (24) zu der durch analytische Fortsetzung allein bzw. normale Integration mit $D^{\text {ret }}$ gelie-

${ }^{9}$ Der nun folgende Teil der Arbeit fußt auf der Dissertation von Bennewitz [31], wo man weitergehende Einzelheiten findet.

${ }^{10}$ Dies soll durch den Balken am Integralzeichen angedeutet werden. Das Zeichen anc bedeutet analytische Fortsetzung in der $\lambda$-Ebene.

${ }^{11}$ In der Regel ist das Integral (23) über jedem der Teilbereiche eine in $\lambda$ meromorphe Funktion, aber die Konvergenzbereiche in der $\lambda$-Ebene sind disjunkt (bis auf höchstens einen Punkt), so daß (23) unzerschnitten fast nirgends konvergiert. 
ferten Lösung ${ }^{12}$. Insofern stellt unser Verfahren eine Erweiterung der Greenschen Methode dar.

Die bei den Einteilchen-Feldgleichungen (2) für beliebige Multipolteilchen auftretenden Feldanteile der Inhomogenitäten (3) haben nun die allgemeine Form

$$
g(x)=\sum_{k} \omega_{\mu_{1} \ldots \mu_{n}}^{(k)}(s) \varrho^{k} R^{\mu_{1}} \ldots R^{\mu_{n}}(\text { ret }),
$$

wobei das übliche Greensche Verfahren nur für $g=\omega \hat{\varrho}^{n}, n \leqq 2$ eine Lösung von (22) liefert. Die Einführung des Parameters $\lambda$ soll nun dadurch erfolgen, daß in den $\hat{h}$-Potentialen erster Näherung die Größe $\hat{\varrho}$ durch $\hat{\varrho}^{\lambda}$ ersetzt wird (was einer formalen Abänderung des Newtonschen Gravitationsgesetzes entspricht). Die Inhomogenität nimmt damit die allgemeine Gestalt

$$
g_{\lambda}(x)=\sum_{k} \omega_{\mu_{1} \ldots \mu_{n}}^{(k, \lambda)}(s) \varrho^{k+\lambda-1} R^{\mu_{1}} \ldots R^{\mu_{n}}(\text { ret })
$$

$a^{13}$, wobei sich die hier rechts auftretende Retardierung im folgenden einfacher handhaben läßt, wenn wir (26) in die Form

$$
g_{\lambda}(x)=\sum_{k} \int_{-\infty}^{\infty} D^{\mathrm{ret}}(x-\xi) \omega_{\mu_{1} \ldots \mu_{n}}^{(k, \hat{n})} \hat{\varrho}^{k+\hat{\lambda}-2} R^{\mu_{1}} \ldots R^{\mu_{n}} d s
$$

setzen.

Die in (23) vorgesehene $y$-Integration läßt sich damit wie folgt durchführen (siehe Anhang):

$$
\varphi_{\lambda}=\underset{i}{\operatorname{anc}} \int_{-\infty}^{s_{\text {ret }}} J_{\lambda} d s
$$

mit

$$
J_{\lambda}=\sum_{k} \omega_{\mu_{1} \ldots \mu_{n}}^{(k, \lambda)} C_{k+\lambda-1}^{\mu_{1} \ldots \mu_{n}}
$$

${ }^{12}$ Die regularisierende Wirkung der getrennten analytischen Fortsetzung eines zerschnittenen Integrals soll an einem einfachen Beispiel aufgezeigt werden: Das über die reelle Achse erstreckte Integral $\int_{a}^{\infty} x^{\lambda} d x$ für beliebiges komplexes $\lambda$ ergibt sich für $a>0$ bereits durch analytische Fortsetzung aus der Halbebene $\operatorname{Re} \lambda \geqq 1$, während für $a \leqq 0$ das Integral irgendwo auf der positiven Halbachse zerschnitten werden muß, für $a=-\infty$ zusätzlich noch irgendwo auf der negativen Halbachse. Dies ergibt dann die Regularisierung

$$
\int_{a}^{\infty} x^{\lambda} d x=\left\{\begin{array}{l}
0 \text { für } a= \pm \infty \text { oder } a=0 \\
-a^{\lambda+1} /(\lambda+1) \text { sonst } .
\end{array}\right.
$$

${ }^{13}$ Zur Vereinfachung der Schreibweise haben wir die Substitution $2 \lambda-1 \rightarrow \lambda$ vorgenommen. 
und $^{14}$

$$
(\lambda-2)(\lambda-3) \ldots(\lambda-n-1) C_{\lambda}^{\mu_{1} \ldots \mu_{n}}=\dot{\nabla}^{\mu_{1}} \ldots \dot{\nabla}^{\mu_{n}} C_{\lambda-n}
$$

$\operatorname{mit}^{15}$

$$
\begin{aligned}
C_{\lambda} & =\frac{1}{4 \pi} \int D^{\mathrm{ret}}(x-y) D^{\mathrm{ret}}(y-\xi) \varrho^{\lambda-1} d y \\
& =\frac{2^{\lambda-3}}{\lambda-2} \frac{\Theta(R)}{\varrho}\left[(u R+\varrho)^{\lambda-2} R^{2(2-\lambda)}-(u R+\varrho)^{2-\lambda}\right] \\
& =\frac{2^{\lambda-3}}{\lambda-2} \frac{\Theta(R)}{\varrho^{\lambda-1}}\left[(k-1)^{2-\lambda}-(\underset{0}{k}+1)^{2-\lambda}\right] .
\end{aligned}
$$

Der Integrand $J_{\lambda}$ hat demnach die allgemeine Form

$$
J_{\lambda}=A_{\lambda} R^{2(1-\lambda)}+\sum_{l=0}^{L} A_{\lambda}^{l} R^{-2(l+\lambda)}
$$

mit an e $s=s_{\text {ret }}\left(R^{2}=0\right)$ regulären Koeffizienten $A_{\lambda}(R, s)$ und $A_{\hat{\lambda}}^{l}(R, s)$. Somit hat die Funktion $\varphi_{\lambda}$ wegen

$$
\begin{aligned}
\underset{\lambda \rightarrow 1}{\operatorname{anc}}(\lambda-1) & \int_{-\infty}^{s_{\mathrm{ret}}} A_{\lambda}^{l} R^{-2(l+\lambda)} d s \\
& =\frac{(-1)^{l+1}}{2^{l+1} l !}\left(\hat{\varrho} \frac{d}{d s}\right)^{l}\left(\varrho A_{1}^{l}\right)(\mathrm{ret}), \quad l=0,1,2, \ldots
\end{aligned}
$$

bei $\lambda=1$ im allgemeinen einen Pol erster Ordnung. Wir können jedoch zeigen, daß die Summe der Residuen (30) dann verschwindet, wenn sich die nicht integrabel singulären Terme in (28c) für $\lambda=1 \mathrm{zu}$ einem totalen Differential zusammenfassen lassen:

$$
J_{1}=A_{1}+\sum_{l=1}^{L}\left(B_{l} R^{-2 l}\right)^{.},
$$

${ }^{14}$ Notation:

$$
\begin{aligned}
\dot{V}_{\alpha} & =\partial / \partial u^{\alpha}, \quad R^{ \pm 2 \lambda}=\left(R^{2}\right)^{ \pm \lambda}, \\
\varrho & =\sqrt{(u R)^{2}-u^{2} R^{2}}, \quad k^{\alpha}=R^{\alpha} / \varrho, \\
k & =u k\left(k_{0}^{2}=1+k^{2}\right), \quad k=\dot{u} k, \quad k=\ddot{u} k .
\end{aligned}
$$

(Von der Normierung $u^{2}=1$ ist erst nach Ausführung der $\dot{V}$-Operationen Gebrauch zu machen. Die Definition des Vektors $k^{\alpha}$ geht für retardierte Eigenzeiten in diejenige von Anmerkung 5 über.)

${ }^{15}$ Für $\lambda \rightarrow 2$ ergibt sich rechts in (29b) der Grenzwert

$$
C_{2}=-\Theta(R) \frac{l}{2 \varrho} \quad \text { mit } \quad l=\ln \frac{\underset{\substack{0 \\ k}+1}{k+1}}{0} \text {. }
$$


wobei für $A_{1}(R, s)$ an der Stelle $s=s_{\text {ret }}$ höchstens eine logarithmische Singularität zugelassen ist, während $B_{l}(R, s)$ dort regulär sein muß.

$\mathrm{Zu}$ diesem Zweck überzeugen wir uns zunächst davon, daß die Hilfsfunktion

$$
\hat{\varphi}_{\lambda}=\int_{-\infty}^{s_{\text {ret }}} J_{1} R^{2(1-\lambda)} d s
$$

an der Stelle $\lambda=1$ regulär ist. Setzt man sie nämlich durch partielle Integration in die Form

$$
\begin{aligned}
\hat{\varphi}_{\lambda}= & \int_{-\infty}^{s_{\text {ret }}}\left[A_{1}+\sum_{l}\left(B_{l} R^{-2 l}\right)^{\cdot}\right] R^{2(1-\lambda)} d s \\
= & \left.\int_{-\infty}^{s_{\text {ret }}} A_{1} R^{2(1-\lambda}\right) d s+\sum_{l} f_{-\infty}^{s_{\text {ret }}}\left(B_{l} R^{2(1-l-\lambda)}\right)^{\cdot} d s \\
& -2(\lambda-1) \sum_{l} f_{-\infty}^{s_{\text {ret }}} B_{l}(u R) R^{-2(l+\lambda)} d s,
\end{aligned}
$$

so ist der erste Term bei $\lambda=1$ regulär:

$$
\underset{\lambda \rightarrow 1}{\operatorname{anc}} \int_{-\infty}^{s_{\text {ret }}} A_{1} R^{2(1-\lambda)} d s=\int_{-\infty}^{s_{\text {ret }}} A_{1} d s
$$

während der zweite Term gemäß der Definition des Operators $\underset{\lambda}{\operatorname{anc} f \text { ver- }}$ schwindet. Schließlich läßt sich das letzte Integral rechts in $\left(32^{\prime}\right)$ mittels (30) nach $\lambda=1$ fortsetzen.

Durch gleichzeitige Addition und Subtraktion der bei $\lambda=1$ regulären Funktionen $\hat{\varphi}_{\lambda}$ können wir nun $\varphi_{\lambda}$ in die folgende Form setzen:

$$
\varphi_{\lambda}=\hat{\varphi}_{\lambda}+\underset{\lambda}{\operatorname{anc}} \int_{-\infty}^{s_{\text {ret }}}\left(J_{\lambda}-J_{1} R^{2(1-\lambda)}\right) d s .
$$

Hier liefert Taylor-Entwicklung des Integranden an der Stelle $\lambda=1$ einen Faktor $(\lambda-1)$, woraufhin sich das Integral mittels (30) analytisch nach $\lambda=1$ fortsetzen $l a ̈ ß t^{16}$ :

$\varphi=-\frac{\hat{\varrho}}{2} A_{1}^{0^{\prime}}+\sum_{l=1}^{L} \frac{(-1)^{l}}{2^{l} l !}\left(\hat{\varrho} \frac{d}{d s}\right)^{l}\left(B_{l}-\frac{\hat{\varrho}}{2} A_{1}^{l \prime}\right)(\mathrm{ret})+\int_{-\infty}^{s_{\mathrm{ret}}} A_{1} d s$.

Ihre Verifizierung vorwegnehmend (siehe die folgenden Beispiele) ist dies die allgemeine Form der Lösung der Einteilchen-Feldgleichungen. Das übliche Greensche Verfahren liefert nur den Integralterm $\int A_{1} d s$ und die divergenten Terme $\left.\Sigma\left(B_{l} R^{-2 l}\right)\right|_{-\infty} ^{s_{\text {ret }}}$. Dabei ist hervorzuheben, daß der Integralterm i.a. selbst dann keine Lösung von (22) mit (25) darstellt,

\footnotetext{
${ }^{16}$ Wir setzen $\varphi_{1}=\varphi$. Der Strich $\left({ }^{\prime}\right)$ bedeutet die Ableitung nach $\lambda$.
} 
wenn die $B_{l}$ in (31) verschwinden (obwohl dann kein Divergenzproblem auftritt). In der Tat ist dann zwar $A_{1}^{l}=0$, jedoch im allgemeinen $A_{1}^{l \prime} \neq 0$.

\subsection{Ergebnisse}

1. Die Lösung der Wellengleichung

$$
\square \varphi=\omega \hat{\varrho}^{2} \text { (ret) }
$$

läßt sich mit der üblichen Greenschen Methode gewinnen und lautet im Hinblick auf (29)

$$
\varphi=-\frac{1}{2} \int_{-\infty}^{s_{\mathrm{ret}}} \frac{\omega l}{\varrho} d s
$$

2. Zur Lösung der Wellengleichung

$$
\square \varphi^{\mu}=\omega_{1} \hat{\varrho}\left(\omega_{2} \varrho\right)^{\mid \mu} \text { (ret) }
$$

muß jedoch bereits das $\lambda$-Schnittverfahren herangezogen werden: Wir ersetzen die Inhomogenität durch $\omega_{1} \hat{\varrho}^{\lambda}\left(\omega_{2} \hat{\varrho}^{\hat{\lambda}}\right)^{\mid \mu}$ (ret) und berechnen (28) (mit $2 \lambda-1 \rightarrow \lambda$ ):

$$
\begin{aligned}
\varphi_{(\lambda)}^{\mu}= & \underset{\lambda}{\operatorname{anc}} \underset{-\infty}{s_{\text {ret }}} \omega_{1}\left\{\frac { \lambda + 1 } { 2 } \omega _ { 2 } \left(u^{\mu} C_{\lambda+2}-C_{\lambda+3}^{\mu}\right.\right. \\
& \left.\left.+\dot{u}_{\nu} C_{\lambda+3}^{\mu v}\right)-\dot{\omega}_{2} C_{\lambda+2}^{\mu}\right\} d s .
\end{aligned}
$$

Mit Hilfe von (29) wird der Integrand durch Abspalten der divergenzerzeugenden $R^{2}$-Potenzen in die Form (28c) gebracht und nach $\lambda=1$ fortgesetzt:

$$
\begin{aligned}
\varphi_{(1)}^{\mu}= & \int_{-\infty}^{s_{\mathrm{ret}}} \frac{\omega_{1}}{\varrho}\left(\omega_{2} A^{\mu}+\dot{\omega}_{2} A^{\mu}\right) d s \\
& +\operatorname{anc}_{\lambda \rightarrow 1}(\lambda-1) \underset{-\infty}{s_{\mathrm{ret}}} \frac{2^{\lambda}}{4 \lambda} \omega_{1} \omega_{2} \varrho^{\lambda-1}(k+1)^{\lambda}\left(u^{\mu}-k^{\mu}\right) R^{-2 \lambda} d s .
\end{aligned}
$$

Hierbei bedeutet

$$
\begin{aligned}
A^{\mu}= & \varrho\left(u^{\mu} C_{3}-C_{4}^{\mu}+\dot{u}_{v} C_{4}^{\mu v}\right) \\
= & k\left[\frac{3}{2} k+\left(\frac{1}{2}+\frac{3}{4} k^{2}\right) l\right] k^{\mu}-\underset{1}{k}\left[\frac{1}{2}+3 k^{2}\left(\frac{1}{2}+\frac{1}{4} k l\right)\right] u^{\mu} \\
& +\left(\frac{1}{2} k+\frac{1}{4} k^{2} l\right) \dot{u}^{\mu}, \\
A^{\mu}= & -\varrho C_{3}^{\mu}=-\left(1+\frac{1}{2} \underset{0}{k} l\right) k^{\mu}+\left(\underset{0}{k}+\frac{1}{2} k^{2} l\right) u^{\mu} .
\end{aligned}
$$

Mit Hilfe von (30) ergibt sich damit die gesuchte Lösung $\varphi^{\mu}=\varphi_{(1)}^{\mu}$ :

$$
\varphi^{\mu}=\frac{\omega_{1} \omega_{2}}{2} \hat{\varrho}\left(k^{\mu}-u^{\mu}\right)(\text { ret })+\int_{-\infty}^{s \text { ret }} \frac{\omega_{1}}{\varrho}\left(\omega_{2} A^{\mu}+\dot{\omega}_{2} A^{\mu}\right) d s,
$$


wobei die Größe (31) die Form

$$
J_{(1)}^{\mu}=\frac{\omega_{1}}{\varrho}\left(\omega_{2} A^{\mu}+\dot{\omega}_{2} A^{\mu}\right)
$$

hat. Das übliche Greensche Verfahren führt hier nicht auf Divergenzen, liefert jedoch nur den Integralterm von (35d), der keine Lösung von (35a) ist.

3. Entsprechend liefert das $\lambda$-Schnittverfahren für die Wellengleichung

die Lösung ${ }^{17}$

$$
\square \varphi^{\mu v}=\left(\omega_{1} \hat{\varrho}\right)^{\mid \mu}\left(\omega_{2} \hat{\varrho}\right)^{\mid v}(\mathrm{ret})
$$

$$
\begin{aligned}
\varphi^{\mu v}= & \hat{\varrho}\left\{\omega _ { 1 } \omega _ { 2 } \left[\left(\frac{1}{4} \hat{\varrho}-\frac{1}{6} k\right) \eta^{\mu v}+\left(\frac{1}{4} \hat{\varrho}-\frac{1}{3} k\right) k^{\mu} k^{v}\right.\right. \\
& -\left(\frac{1}{4} \hat{\varrho}-\frac{1}{12} k\right) u^{(\mu} k^{v)}+\frac{1}{3} k u^{\mu} u^{v}+\frac{1}{12} \dot{u}^{(\mu} k^{v)} \\
& \left.-\frac{1}{3} u^{(\mu} \dot{u}^{v)}\right]+\left(\omega_{1} \omega_{2}\right)^{\cdot}\left[\frac{1}{12} \eta^{\mu v}+\frac{1}{4} k^{\mu} k^{v}+\frac{1}{4} u^{(\mu} k^{v)}\right. \\
& \left.\left.-\frac{1}{3} u^{\mu} u^{\nu}\right]-\frac{1}{2}\left(\omega_{1} \dot{\omega}_{2} u^{\mu} k^{\nu}+\dot{\omega}_{1} \omega_{2} k^{\mu} u^{v}\right)\right\}(\mathrm{ret}) \\
& -\frac{1}{8} \int_{-\infty}^{s_{\text {ret }}} \frac{1}{\varrho}\left[\omega_{1} \omega_{2} A^{\mu v}+\left(\omega_{1} \omega_{2}\right)^{\cdot} A^{\mu v}+\dot{\omega}_{1} \dot{\omega}_{2} A^{\mu \nu}\right] d s
\end{aligned}
$$

mit

$$
\begin{aligned}
& A^{\mu v}=8 \varrho \dot{u}_{\varrho} \dot{u}_{\sigma} C_{6}^{\mu \nu \varrho \sigma}=\eta^{\mu v}\left\{\left[k_{1}^{2}\left(2+\frac{5}{2} k^{2}\right)+\frac{1}{2} w k^{2}\right] k^{2} l\right. \\
& \left.+\underset{0}{k k_{1}^{2}}\left(\frac{2}{3}+5 k^{2}\right)-w k\left(\frac{2}{3}-k^{2}\right)\right\}+k^{\mu} k^{\nu}\left\{\left[k_{1}^{2}\left(4+20 k^{2}+\frac{35}{2} k^{4}\right)\right.\right. \\
& \left.\left.+w k^{2}\left(2+\frac{5}{2} k^{2}\right)\right] l+\underset{0}{k k_{1}^{2}}\left(\frac{50}{3}+35 k^{2}\right)+w k\left(\frac{2}{3}+5 k^{2}\right)\right\} \\
& +u^{(\mu} k^{v)}\left\{-\left[k_{1}^{2}\left(10+\frac{35}{2} k^{2}\right)+\frac{5}{2} w k^{2}\right] k k^{2} l-k_{1}^{2}\left(2+\frac{95}{3} k^{2}+35 k^{4}\right)\right. \\
& \left.+w\left(\frac{2}{3}-\frac{5}{3} k^{2}-5 k^{4}\right)\right\}+\dot{u}^{(\mu} k^{\nu)} k\left\{\left(4+5 k^{2}\right) k^{2} l+k_{0}\left(\frac{4}{3}+10 k^{2}\right)\right\} \\
& +u^{\mu} u^{v}\left\{\left[k_{1}^{2}\left(15+\frac{35}{2} k^{2}\right)+\frac{5}{2} w k^{2}\right] k^{4} l+\underset{0}{k k_{1}^{2}}\left(-\frac{4}{3}+\frac{20}{3} k^{2}+35 k^{4}\right)\right. \\
& \left.+w k\left(\frac{8}{3}-\frac{10}{3} k^{2}+5 k^{4}\right)\right\}+u^{(\mu} \dot{u}^{v)} k_{1}\left\{-5 k k^{4} l+\frac{4}{3}-\frac{10}{3} k^{2}-10 k^{4}\right\} \\
& +\dot{u}^{\mu} \dot{u}^{v}\left\{k^{4} l-\underset{0}{k}\left(\frac{4}{3}-2 k^{2}\right)\right\} \text {, } \\
& A^{\mu \nu}=-8 \varrho \dot{u}_{\varrho} C_{5}^{\mu \nu} \varrho=-2 \eta^{\mu v} k\left\{\frac{2}{3}+2 k^{2}+k k^{2} l\right\}-4 k^{\mu} k^{v} k\left\{\frac{11}{3}+5 k^{2}\right. \\
& \left.+k_{0}\left(1+\frac{5}{2} k^{2}\right) l\right\}+4 u^{(\mu} k^{v)} \underset{1}{k}\left\{k\left(\frac{2}{3}+5 k^{2}\right)+\left(2+\frac{5}{2} k^{2}\right) k^{2} l\right\} \\
& -2 \dot{u}^{(\mu} k^{\nu)}\left\{\frac{2}{3}+2 k^{2}+k_{0} k^{2} l\right\}+4 u^{\mu} u^{v} k\left\{\frac{2}{3}-\frac{5}{3} k^{2}-5 k^{4}-\frac{5}{2}_{0} k k^{4} l\right\} \\
& -2 u^{(\mu} \dot{u}^{v)}\left\{k\left(\frac{4}{3}-2 k^{2}\right)-k^{4} l\right\} \text {, } \\
& A^{\mu v}=8 \varrho C_{4}^{\mu v}=2 \eta^{\mu v}\left\{2 \underset{0}{2 k}+k^{2} l\right\}+2 k^{\mu} k^{v}\left\{6 \underset{0}{6}+\left(2+3 k^{2}\right) l\right\} \\
& -2 u^{(\mu} k^{v)}\left\{2+6 k^{2}+3 k k^{2} l\right\}-2 u^{\mu} u^{v}\left\{k\left(4-6 k^{2}\right)-3 k^{4} l\right\} \text {. }
\end{aligned}
$$

Die Größe (31) lautet hier

$$
J_{(1)}^{\mu \nu}=-\frac{1}{8 \varrho}\left[\omega_{1} \omega_{2} A^{\mu \nu}+\left(\omega_{1} \omega_{2}\right)^{\cdot} A^{\mu \nu}+\dot{\omega}_{1} \dot{\omega}_{2} A^{\mu \nu}\right]+\frac{2}{3}\left(\omega_{1} \omega_{2} p^{\mu \nu} / R^{2}\right)^{.}
$$

und führt beim herkömmlichen Greenschen Verfahren auf Divergenzen.

\footnotetext{
17 Notation: $w=\dot{u}^{2}$
} 
4. Schließlich ergibt sich für die Wellengleichung

die Lösung

$$
\square \varphi^{\mu v}=\omega_{1} \hat{\varrho}\left(\omega_{2} \hat{\varrho}\right)^{\mid \mu v}(\mathrm{ret})
$$

$$
\begin{aligned}
\varphi^{\mu \nu}= & \hat{\varrho}\left\{\omega _ { 1 } \omega _ { 2 } \left[\frac{1}{4} \varrho \eta^{\mu \nu}+\left(\frac{3}{4} \hat{\varrho}-k\right) k_{1}^{\mu} k^{\nu}-\left(\frac{3}{4} \hat{\varrho}-\frac{1}{4} k\right) u^{(\mu} k^{v)}\right.\right. \\
& \left.-\frac{1}{4} \dot{u}^{(\mu} k^{v)}+\frac{1}{2} \varrho u^{\mu} u^{v}\right]+\omega_{1} \dot{\omega}_{2}\left[\frac{1}{2} \eta^{\mu \nu}+\frac{1}{2} k^{\mu} k^{\nu}-u^{\mu} u^{\nu}\right] \\
& \left.+\left(\omega_{1} \omega_{2}\right)^{v}\left[-\frac{1}{12} \eta^{\mu \nu}+\frac{1}{4} k^{\mu} k^{\nu}-\frac{1}{4} u^{(\mu} k^{\nu)}+\frac{1}{3} u^{\mu} u^{\nu}\right]\right\}(\mathrm{ret}) \quad(37 \mathrm{~b}) \\
& +\int_{-\infty}^{s_{\mathrm{ret}}} \frac{\omega_{1}}{\varrho}\left(\omega_{2} A^{\mu v}+\dot{\omega}_{2} A^{\mu \nu}+\ddot{\omega}_{2} A^{\mu \nu}\right) d s .
\end{aligned}
$$

Dabei ist:

$$
\begin{aligned}
& A^{\mu \nu}=\varrho\left(-3 \dot{u}_{\varrho} \dot{u}_{\sigma} C_{6}^{\mu \nu \rho \sigma}+\ddot{u}_{\varrho} C_{5}^{\mu \nu \rho}\right)=\eta^{\mu \nu}\left\{\left[-k^{2}\left(\frac{3}{4}+\frac{15}{16} k^{2}\right)\right.\right. \\
& \left.-\frac{3}{16} w k^{2}+\frac{1}{4} k k\right] k^{2} l-k_{0} k^{2}\left(\frac{1}{4}+\frac{15}{8} k^{2}\right)+w k\left(\frac{1}{4}-\frac{3}{8} k^{2}\right) \\
& \left.+k\left(\frac{1}{6}+\frac{1}{2} k^{2}\right)\right\}+k^{\mu} k^{v}\left\{\left[-k^{2}\left(\frac{3}{2}+\frac{15}{2} k^{2}+\frac{105}{16} k^{4}\right)\right.\right. \\
& \left.-w k^{2}\left(\frac{3}{4}+\frac{15}{16} k^{2}\right)+k k\left(\frac{1}{2}+\frac{5}{4} k^{2}\right)\right] l-k k^{2}\left(\frac{25}{4}+\frac{105}{8} k^{2}\right) \\
& \left.-w k\left(\frac{1}{4}+\frac{15}{8} k^{2}\right)+k\left(\frac{11}{6}+\frac{5}{2} k^{2}\right)\right\}+u^{(\mu} k^{v)}\left\{\left[k_{0} k^{2}\left(\frac{15}{4}+\frac{105}{16} k^{2}\right)\right.\right. \\
& \left.\left.+\frac{15}{16} w k k^{2}-k\left(1+\frac{5}{4} k^{2}\right)\right] k^{2} l+k^{2}\left(\frac{3}{4}+\frac{95}{8} k^{2}+\frac{105}{8}\right) k^{4}\right) \\
& \left.+w\left(-\frac{1}{4}+\frac{5}{8} k^{2}+\frac{15}{8} k^{4}\right)-{ }_{02} k\left(\frac{1}{3}+\frac{5}{2} k^{2}\right)\right\} \\
& -\dot{u}^{(\mu} k^{\nu)}\left\{k\left(\frac{3}{2}+\frac{15}{8} k^{2}\right) k^{2} l+k_{0} k\left(\frac{1}{2}+\frac{15}{4} k^{2}\right)\right\}+\ddot{u}^{(\mu} k^{\nu)}\left\{\frac{1}{4} k k^{2} l+\frac{1}{6}+\frac{1}{2} k^{2}\right\} \\
& -u^{\mu} u^{v}\left\{\left[k^{2}\left(\frac{45}{8}+\frac{105}{16} k^{2}\right)+\frac{15}{16} w k^{2}-\frac{5}{4} k k\right] k^{4} l\right. \\
& -k k^{2}\left(\frac{1}{2}-\frac{5}{2} k^{2}-\frac{105}{8} k^{4}\right)+w k\left(1-\frac{5}{4} k^{2}+\frac{15}{8} k^{4}\right) \\
& \left.+k\left(\frac{1}{3}-\frac{5}{6} k^{2}-\frac{5}{2} k^{4}\right)\right\}+u^{(\mu} \dot{u}^{\nu)}\left\{\frac{15}{8} k k k^{4} l\right. \\
& \left.+k\left(-\frac{1}{2}+\frac{5}{4} k^{2}+\frac{15}{4} k^{4}\right)\right\}+u^{(\mu} \ddot{u}^{\nu)}\left\{-\frac{1}{4} k^{4} l+k\left(\frac{1}{3}-\frac{1}{2} k^{2}\right)\right\} \\
& +\dot{u}^{\mu} \dot{u}^{v}\left\{-\frac{3}{8} k^{4} l+\underset{0}{k}\left(\frac{1}{2}-\frac{3}{4} k^{2}\right)\right\}, \\
& A^{\mu \nu}=3 \varrho \dot{u}_{\varrho} C_{5}^{\mu v \varrho}=\eta^{\mu v} k\left\{\frac{3}{4} k k^{2} l+\frac{1}{2}+\frac{3}{2} k^{2}\right\} \\
& +k^{\mu} k_{1}^{v} k\left\{k\left(\frac{3}{2}+\frac{15}{4} k^{2}\right) l+\frac{11}{2}+\frac{15}{2} k^{2}\right\}-u^{(\mu} k_{1}^{\nu)} k\left\{\left(3+\frac{15}{4} k^{2}\right) k^{2} l\right. \\
& \left.+k\left(1+\frac{15}{2} k^{2}\right)\right\}+\dot{u}^{(\mu} k^{v)}\left\{\frac{3}{4} k k^{2} l+\frac{1}{2}+\frac{3}{2} k^{2}\right\} \\
& +u^{\mu} u^{v} k\left\{\frac{15}{4} k k^{4} l-1+\frac{5}{2} k^{2}+\frac{15}{2} k^{4}\right\}+u^{(\mu} \dot{u}^{\nu)}\left\{-\frac{3}{4} k^{4} l+\underset{0}{k}\left(1-\frac{3}{2} k^{2}\right)\right\} \text {, } \\
& A^{\mu v}=-\varrho C_{4}^{\mu v}=-\eta^{\mu \nu}\left\{\frac{1}{4} k^{2} l+\frac{1}{2} k\right\}-k^{\mu} k^{v}\left\{\left(\frac{1}{2}+\frac{3}{4} k^{2}\right) l+\frac{3}{2} k\right\} \\
& +u^{(\mu} k^{v)}\left\{\frac{3}{4} k k^{2} l+\frac{1}{2}+\frac{3}{2} k^{2}\right\}+u^{\mu} u^{v}\left\{-\frac{3}{4} k^{4} l+\underset{0}{k}\left(1-\frac{3}{2} k^{2}\right)\right\}
\end{aligned}
$$

Die Größe (31) hat die Form

$$
J_{(1)}^{\mu v}=\frac{\omega_{1}}{\varrho}\left(\omega_{2} A^{\mu v}+\dot{\omega}_{2} A^{\mu v}+\ddot{\omega}_{2} A^{\mu \nu}\right)
$$

und das Greensche Verfahren liefert den Integralterm ohne Divergenzen, jedoch keine Lösung. 
Sämtliche hier angegebenen Lösungen lassen sich durch direkte Anwendung des $\square$-Operators verifizieren ${ }^{18}$. Bei der Berechnung von Spinteilchen-Potentialen zweiter Näherung treten auch noch Inhomogenitäten auf, bei denen die Summe der Ableitungen bis vier läuft.

\section{Zweite Näherung}

\subsection{Das Polteilchen}

Für ein einzelnes Polteilchen haben die Feldgleichungen in zweiter Näherung gemäß (17) folgende Gestalt:

$$
\begin{aligned}
\square \hat{h}_{\alpha}^{\beta}=- & 16 \pi \alpha \int_{-\infty}^{\infty} u_{\alpha} u^{\beta} \delta(R) d s \\
& -4 \alpha^{2} \hat{\varrho}^{4}\left(\frac{1}{2} \delta_{\alpha}^{\beta}+3 u_{\alpha} u^{\beta}-u_{(\alpha} k^{\beta)}+k_{\alpha} k^{\beta}\right) \text { (ret), }
\end{aligned}
$$

wobei wir durchgehend von den Bewegungsgleichungen in nullter und erster Näherung $\left(m=m_{0}, \dot{u}^{\alpha}=0\right)$ Gebrauch machen ${ }^{19}$. Anstelle des divergenten Ergebnisses der Greenschen Methode liefert das $\lambda$-Schnittverfahren folgende retardierte Lösung von (38):

$$
\hat{h}_{\alpha}^{\beta}=-4 \alpha \varrho u_{\alpha} u^{\beta}-\alpha^{2} \hat{\varrho}^{2}\left(8 u_{\alpha} u^{\beta}-u_{(\alpha} k^{\beta)}+k_{\alpha} k^{\beta}\right) \text { (ret). }
$$

Sie erfüllt zugleich die Bedingung $\hat{h}_{\alpha \mid \beta}^{\beta}=0$. Die zugehörige Metrik (1b) ergibt sich damit über (4) in der Form

$$
g_{\alpha \beta}=\eta_{\alpha \beta}+2 \alpha \hat{\varrho}\left(\eta_{\alpha \beta}-2 u_{\alpha} u_{\beta}\right)+\alpha^{2} \hat{\varrho}^{2}\left(\eta_{\alpha \beta}+u_{(\alpha} k_{\beta)}-k_{\alpha} k_{\beta}\right) \text { (ret) . }
$$

Dies ist nichts anderes als die zweite Näherung der Metrik $(\varrho=1 / \varrho=u R)$ :

$g_{\alpha \beta}=\left(1+\frac{\alpha}{\varrho}\right)^{2} \eta_{\alpha \beta}-\frac{4 \alpha \varrho}{\varrho^{2}-\alpha^{2}} u_{\alpha} u_{\beta}+\frac{\alpha^{2}}{\varrho^{2}} \frac{\varrho+\alpha}{\varrho-\alpha}\left(u_{(\alpha} k_{\beta)}-k_{\alpha} k_{\beta}\right)(\mathrm{ret})$,

die ihrerseits im Ruhsystem in die (auf harmonische Polar-Koordinaten ${ }^{20}$ bezogene) Schwarzschildsche Maßbestimmung

$$
d \sigma^{2}=\frac{\varrho-\alpha}{\varrho+\alpha} d t^{2}-\frac{\varrho+\alpha}{\varrho-\alpha} d \varrho^{2}-(\varrho+\alpha)^{2}\left(d \vartheta^{2}+\sin ^{2} \vartheta d \varphi^{2}\right)
$$

übergeht (vgl. [32]).

18 Dabei ist von der Formel

$\square \int_{-\infty}^{s_{\text {ret }}} A(R, s) d s=\int_{-\infty}^{s_{\text {ret }}} \square A(R, s) d s+2 \lim _{s \rightarrow s_{\text {ret }}} \hat{\varrho}\left(1+R_{\text {ret }}^{\alpha} \partial_{\alpha}\right) A(R, s)$.

Gebrauch zu machen.

19 Mit den Ergebnissen von $\S 3$ läßt sich auch leicht die entsprechende Lösung für ein beliebig bewegtes Polteilchen gewinnen, der jedoch keine physikalische Bedeutung zukommt, da sie die Bedingung $\hat{h}_{\alpha \mid \beta}^{\beta}=0$ verletzt (siehe jedoch den abweichenden Standpunkt in [10]).

${ }^{20} R_{\mathrm{ret}}^{\alpha}=\varrho(1, \sin \vartheta \cos \varphi, \sin \vartheta \sin \varphi, \cos \vartheta), \xi^{\alpha}=(t, 0,0,0)$. 
Um den Einfluß des gravitativen Eigenfeldes auf die Bewegung des Polteilchens in zweiter Näherung zu untersuchen, müßen wir die Lösung von (38) und ihre Ableitungen am Ort des Teilchens berechnen und in die Bewegungsgleichungen (7) einsetzen. $\mathrm{Zu}$ diesem Zweck definieren wir in Analogie zu [27] das Eigenpotential am Ort der Quelle durch folgenden Grenzprozeß

$$
\hat{h}^{\alpha \beta}(\xi)=\frac{1}{2} \lim _{x \rightarrow \xi}\left[\hat{h}_{\mathrm{ret}}^{\alpha \beta}(x)-\hat{h}_{\mathrm{adv}}^{\alpha \beta}(x)\right]
$$

(wobei der Feldpunkt $x$ orthogonal zur Weltlinie gegen den Teilchenort $\xi$ konvergieren soll) und entsprechend für die Ableitungen.

Die Übertragung des in $\S 3$ entwickelten Verfahrens auf die avancierte Lösung von (38) liefert nun

$$
\hat{h}_{\mathrm{adv}}^{\alpha \beta}=4 \alpha \varrho u^{\alpha} u^{\beta}-\alpha^{2} \hat{\varrho}^{2}\left(8 u^{\alpha} u^{\beta}-u^{(\alpha} k^{\beta)}+k^{\alpha} k^{\beta}\right)(\mathrm{adv}),
$$

womit der Grenzübergang (41) auch in zweiter Näherung zu dem Ergebnis

$$
\hat{h}_{\alpha}^{\beta}(\xi)=0, \quad \hat{h}_{\alpha \mid \gamma}^{\beta}(\xi)=0
$$

führt. Die damit aus (7) folgenden Bewegungsgleichungen zweiter Näherung

$$
m=m_{0}, \quad \dot{u}^{\alpha}=0
$$

bringen wieder das Fehlen gravitativer Selbstkräfte zum Ausdruck. Diese (störungstheoretische) Konsequenz der Einsteinschen Feldgleichungen steht im Gegensatz zu der entsprechenden Situation in der linearen Elektrodynamik, wo die Selbstbeschleunigung einer Punktladung durch eine Zusatzbedingung eleminiert werden muß [27,28] (die allerdings darauf hinausläuft, daß sich die Lösung nach der Ladung entwickeln läßt).

\subsection{Mehrere Polteilchen}

Das Potential des Mehrteilchenproblems hat jetzt anstelle von (19) die explizite Form

$$
\begin{aligned}
\hat{h}_{\alpha}^{\beta}= & -\sum_{\xi}\left[4 \alpha \hat{\varrho} u_{\alpha} u^{\beta}+\alpha^{2} \hat{\varrho}^{2}\left(8 u_{\alpha} u^{\beta}-u_{(\alpha} k^{\beta)}+k_{\alpha} k^{\beta}\right)\right](\text { ret }) \\
& -\sum_{\xi \bar{\xi}}^{\prime} \alpha \bar{\alpha}\left\{4 \hat{\varrho} \hat{\varrho}\left(1+2 \bar{u}_{0}^{2}\right) u_{\alpha} u^{\beta}\right. \\
& \left.-2 \int_{-\infty}^{\infty} d s \int_{-\infty}^{\infty} d \bar{s} \nabla_{\alpha}^{\beta} \Theta(R) \Theta(\bar{R}) \Theta\left(-|R-\bar{R}|^{2}\right)\left[(R \bar{R})^{2}-R^{2} \bar{R}^{2}\right]^{-\frac{1}{2}}\right\} .
\end{aligned}
$$


Eine eingehendere Diskussion der Weltlinien-Doppelintegrale steht noch aus. Auf den ersten Blick kommt durch sie eine Ausbreitung der Gravitationswirkung (zweiter Näherung) mit beliebigen Unterlichtgeschwindigkeiten zum Ausdruck. Sie könnte durch eine kontinuierliche Rückstreuung von Gravitationsstrahlung an der Energie-Impulsverteilung des Feldes erster Näherung gedeutet werden. Dieser Zweiteilcheneffekt wird nur von kausal nicht korrelierten Teilchenpaaren ausgeübt, das heißt, für $(R-\bar{R})^{2}<0$. Ad hoc-Wechselwirkungen dieses Typs sind kürzlich von Van Dam u. Wigner [33, 34] eingehend diskutiert worden.

\subsection{Das Spinteilchen}

Im Gegensatz zum Polteilchenfeld tritt Selbststreuung von Gravitationsstrahlung bereits im Feld eines einzelnen Spinteilchens auf. Um dies zu zeigen, ist es jedoch nicht nötig, die Feldgleichungen bis zur zweiten Näherung vollständig zu integrieren, obwohl dies mit dem Verfahren von $\S 3$ keine grundsätzlichen Schwierigkeiten mehr bereitet. In der Tat kommt die kontinuierliche Rückstreuung der von einem beliebig bewegten Spinteilchen ausgehenden Gravitationsstrahlung in dem Linienintegral der allgemeinen Lösung (33) zum Ausdruck. (Im Hinblick auf den Formalismus der zweiten Näherung kann man auch sagen, daß die lichtartig von den Punkten der Teilchenweltlinie ausgehende Wirkung durch fiktive Zentren gestreut wird, die kontinuierlich über den Aufpunkt-Vorkegel verteilt sind. Die Gesamtwirkung ergibt sich durch Integration über den in diesem Vorkegel liegenden Weltlinienast.) Es genügt daher, zu zeigen, daß dieses Linienintegral i.a. nicht verschwindet.

$\mathrm{Zu}$ diesem Zweck integrieren wir die Feldgleichungen (2a) über eine beliebige raumartige Hyperfläche $\sigma$, deren Rand $\partial \sigma$ durch den Schnitt mit einem Lichtkegel gebildet werde, der von einem beliebigen Punkt der Weltlinie in der Vergangenheit von $\sigma$ ausgeht (s. Abb. 3) ${ }^{21}$ :

$$
\oint_{\partial \sigma} \hat{h}_{\alpha}^{\beta \mid \gamma} d \sigma_{\beta \gamma}=-\chi \int_{\sigma} \Theta_{\alpha}^{\beta} d \sigma_{\beta} .
$$

Wegen $\Theta_{\alpha \mid \beta}^{\beta}=0$ ist nun das Integral (Gesamtimpuls)

$$
P_{\alpha}^{\text {tot }}=\int_{\sigma_{\infty}} \Theta_{\alpha}^{\beta} d \sigma_{\beta},
$$

${ }^{21}$ Das Oberflächenintegral linker Hand ist hierbei eine Folge des Integralsatzes

$$
\int_{\sigma} d \sigma_{[\alpha} \partial_{\beta]} A=2 \oint_{\partial \sigma} d \sigma_{\alpha \beta} A \text {. }
$$


erstreckt über eine unendliche raumartige Hyperfläche $\sigma_{\infty}=\sigma \cup \bar{\sigma}$ von Gestalt und Lage dieser Hyperfläche tatsächlich unabhängig ${ }^{22}$ Im Hinblick darauf setzen wir (46) in die Form

$$
-\varkappa^{-1} \oint_{\partial \sigma} \hat{h}_{\alpha}^{\beta \mid \gamma} d \sigma_{\beta \gamma}=P_{\alpha}^{\text {tot }}-\int_{\bar{\sigma}} \Theta_{\alpha}^{\beta} d \sigma_{\beta}
$$

und verlegen die Fläche $\partial \sigma$ in die Wellenzone der retardierten Lösung der Feldgleichungen erster Ordnung:

$$
\square \hat{h}_{\alpha}^{\beta}(x)=-\chi \int_{-\infty}^{\infty} u_{(\alpha}\left(P^{\beta)}+S^{\beta) \gamma} \partial \gamma\right) \delta(x-\xi) d s, \quad \hat{h}_{\alpha \mid \beta}^{\beta}=0,
$$

d. h.

$$
\hat{h}_{\alpha}^{\beta}=-\frac{x}{4 \pi} \hat{\varrho}\left[u_{(\alpha} P^{\beta)}+\left(u_{(\alpha} S^{\beta) \gamma} k_{\gamma}\right)\right](\text { ret })
$$

mit

$$
\dot{P}_{\alpha}=0, \quad u_{[\alpha} P_{\beta]}+\dot{S}_{\alpha \beta}=0 .
$$

In der Wellenzone $(\hat{\varrho} \rightarrow 0)$ gilt nun asymptotisch (modulo einer Eichtransformation, vgl. [29]):

$$
\Theta_{\alpha \beta} \rightarrow t_{\alpha \beta}^{\mathrm{rad}},
$$

wobei $t_{\alpha \beta}^{\text {rad }}$ der asymptotisch eichinvariante Energie-Impulstensor des von einem Spinteilchen erzeugten gravitativen Strahlungsfeldes ist (d. h. derjenige Anteil von $\Theta_{\alpha \beta}$, der proportional zu $\hat{\varrho}^{2}$ ist). Er ist im ganzen Feldraum definiert und dort überall quellenfrei und lichtartig (vgl. [12]):

$$
\partial^{\beta} t_{\alpha \beta}^{\mathrm{rad}}=0=k^{\beta} t_{\alpha \beta}^{\mathrm{rad}} \quad(x \neq \xi) .
$$

Damit läßt sich das $\bar{\sigma}$-Integral in (46') über den Gaußschen Satz wie folgt umformen

mit

$$
\int_{\bar{\sigma}} \Theta_{\alpha}^{\beta} d \sigma_{\beta}=-\int_{-\infty}^{s \mathrm{ret}} Y_{\alpha} d s
$$

$$
Y_{\alpha}=\oint t_{\alpha \beta}^{\mathrm{rad}} n^{\beta} d \Omega .
$$

Die Integration in (51) erfolgt über den Schnitt eines vom Teilchen ausgehenden Lichtkegels mit einer seine Weltlinie schlauchförmig umgebenden (zeitartigen) Hyperfläche $\Sigma$. Ferner ist $n^{\beta}$ die (nach außen gerichtete, raumartige) Normale von $\Sigma$ und $d \Omega$ ein Element der Schnittfläche. Zur Begründung betrachten wir die geschlossene Hyperfläche,

${ }^{22}$ Dabei ist vorauszusetzen, daß das Spinteilchen nur während einer endlichen Zeitspanne strahlt: Es soll im räumlich Unendlichen stets eine Kugelfläche geben, die noch nicht von der Strahlung (erster Näherung) erreicht worden ist, falls ihr Radius nur groß genug gewählt wird. 


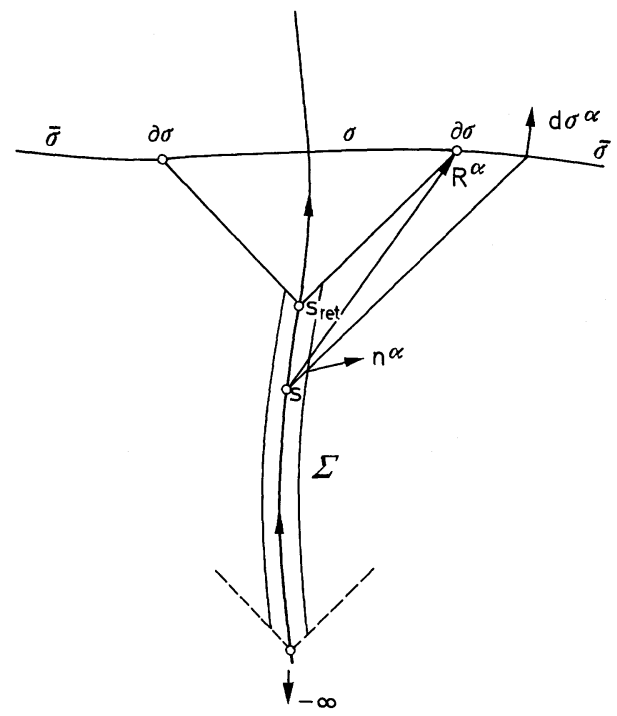

Abb. 3. Zur Selbststreuung der von einem Spinteilchen erzeugten Gravitationsstrahlung

die von $\bar{\sigma}$ und $\Sigma$ sowie den von $s=s_{\text {ret }}$ und $s=-\infty$ ausgehenden Lichtkegeln gebildet wird (s. Abb. 3). Das über diese Hyperfläche erstreckte Integral $\int t_{\alpha \beta}^{\mathrm{rad}} d \sigma^{\beta}$ verschwindet wegen der Quellenfreiheit von $t_{\alpha \beta}^{\mathrm{rad}}$. Andererseits gilt auf den Lichtkegeln $t_{\alpha \beta}^{\mathrm{rad}} d \sigma^{\beta}=0$, da dort $d \sigma^{\beta} \sim k^{\beta}\left(k^{2}=0\right)$ und $t_{\alpha \beta}^{\text {rad }}$ lichtartig ist.

Ferner ist $Y_{\alpha}$ von $\Sigma$ unabhängig, d. h. es hat für alle auf einem festen Lichtkegel gelegene Hüllflächen den gleichen Wert. Damit ist $Y_{\alpha}$ auch eichinvariant, da wir die Hüllfläche auf einem festen Lichtkegel in die Wellenzone verschieben können, wo $t_{\alpha \beta}^{\mathrm{rad}}$ von den Eichtransformationen (5) (in der entsprechenden Ordnung) nicht beeinflußt wird [29]. Eine explizite Darstellung des Strahlungsverlustes $-Y_{\alpha}$ durch Teilchenvariable findet sich in Gl. (30a) von Ref. [12].

Zusammenfassend gelangen wir zu dem Ergebnis

$$
-\chi^{-1} \oint_{\partial \sigma} \hat{h}_{\alpha}^{\beta \mid \gamma} d \sigma_{\beta \gamma}=P_{\alpha}^{\text {tot }}+\int_{-\infty}^{s_{\text {ret }}} Y_{\alpha} d s
$$

wobei die Hüllintegration linker Hand bei festem $s=s_{\text {ret }} \mathrm{zu}$ erfolgen hat. Das Weltlinienintegral rechter Hand ist somit ein Bestandteil der zweiten Näherung $\hat{h}_{\alpha}^{\beta}(x)$ selbst und kann als Manifestation der Selbststreuung von Gravitationsstrahlung aufgefaßt werden. Analoge Verhältnisse treten auch bei anderen Quellverteilungen auf [17, 20, 23-25]. 


\section{Anhang}

\section{Kovariante y-Integration}

1. Das Integral (29b) des Textes, d. h.

$$
C_{\lambda}=\frac{1}{4 \pi} \int D^{\mathrm{ret}}(x-y) D^{\mathrm{ret}}(y-\xi)[u(y-\xi)]^{1-\lambda} d y
$$

mit

$$
D^{\mathrm{ret}}(x)=2 \Theta(x) \delta\left(x^{2}\right), \quad u=\dot{\xi}
$$

ist im $y$-Raum über den Durchschnitt des Nachkegels von $\xi$ mit dem Vorkegel von $x$ zu erstrecken (Abb. 1). Wir wählen den Vektor $R=x-\xi$ als Zeitachse (Zeitkoordinate $\tau$ ) und führen im Orthogonalraum von $R$ Polarkoordinaten $r, \vartheta, \varphi$ ein. Aus

$$
(x-y)^{2}=\tau^{2} R^{2}-r^{2}=0,(y-\xi)^{2}=(\tau+1)^{2} R^{2}-r^{2}=0
$$

folgt $\tau=-\frac{1}{2}$ und $r=\frac{1}{2} \sqrt{R^{2}}$. Wegen

$$
\begin{aligned}
u(y-\xi) & =(\tau+1) u R-r \varrho \cos \vartheta / \sqrt{R^{2}}, \\
\varrho & =\sqrt{(u R)^{2}-u^{2} R^{2}}, d y=\sqrt{R^{2}} r^{2} \sin \vartheta d \tau d r d \vartheta d \varphi
\end{aligned}
$$

reduziert sich das Integral (A1) auf

$$
\begin{aligned}
C_{\lambda} & =2^{\lambda-3} \Theta(R) \int_{0}^{\pi}(u R-\varrho \cos \vartheta)^{1-\lambda} \sin \vartheta d \vartheta \\
& =\frac{2^{\lambda-3}}{\lambda-2} \frac{\Theta(R)}{\varrho}\left[(u R-\varrho)^{2-\lambda}-(u R+\varrho)^{2-\lambda}\right] .
\end{aligned}
$$

2. Der Integrationsbereich des Integrales (21) des Textes, d. h.

$$
D=\frac{1}{4 \pi} \int D^{\mathrm{ret}}(x-y) D^{\mathrm{ret}}(y-\xi) D^{\mathrm{ret}}(y-\bar{\xi}) d y
$$

wird durch den Durchschnitt dreier Halbkegel gebildet: Vorkegel von $x$ und Nachkegel von $\xi$ und $\bar{\xi}$ (Abb. 2). Wir spannen den $y$-Raum jetzt durch die beiden zeitartigen Vektoren $R=x-\xi$ und $\bar{R}=x-\bar{\xi}$ sowie die zu ihnen orthogonale (raumartige) Ebene durch den Punkt $x$ auf, in der wir Polarkoordinaten $r, \varphi$ einführen:

$$
x-y=\tau R+\bar{\tau} \bar{R}+\varepsilon_{1} r \sin \varphi+\varepsilon_{2} r \cos \varphi
$$

( $\varepsilon_{1}$ und $\varepsilon_{2}$ sind beliebige zueinander und zu $R$ und $\bar{R}$ orthogonale, raumartige Einheitsvektoren). Da nun der (positiv genommene) Flächeninhalt des von $R$ und $\bar{R}$ aufgespannten Parallelogramms durch

$$
\sigma=\sqrt{(R \bar{R})^{2}-R^{2} \bar{R}^{2}}
$$


gegeben ist, so gilt für das Volumenelement des $y$-Raumes

$$
d y=\sigma r \sin \varphi d \tau d \bar{\tau} d r d \varphi .
$$

Die Variablentransformation $\tau, \bar{\tau}, r \rightarrow u, v, \bar{v}$ gemä

$$
\begin{aligned}
& u=(x-y)^{2}=\tau^{2} R^{2}+\bar{\tau}^{2} \bar{R}^{2}+2 \tau \bar{\tau} R \bar{R}-r^{2}, \\
& v=(y-\xi)^{2}=(1-\tau)^{2} R^{2}+\bar{\tau}^{2} \bar{R}^{2}-2(1-\tau) \bar{\tau} R \bar{R}-r^{2}, \\
& \bar{v}=(y-\bar{\xi})^{2}=(1-\bar{\tau})^{2} \bar{R}^{2}+\tau^{2} R^{2}-2(1-\bar{\tau}) \tau R \bar{R}-r^{2}
\end{aligned}
$$

führt dann wegen

sofort auf

$$
d y=\frac{1}{8 \sigma} d u d v d \bar{v} d \varphi
$$

$$
D=\frac{1}{2 \sigma} \Theta(R) \Theta(\bar{R}) \Theta\left(-|R-\bar{R}|^{2}\right) .
$$

Hierbeit ergeben sich die $\Theta$-Faktoren aus der Tatsache, daß $D$ gemäß(A3) nur dann nicht verschwindet, wenn $R$ und $\bar{R}$ in oder auf dem Vorkegel von $x$ liegen und $\xi-\bar{\xi}$ nicht zeitartig ist.

Die Verfahren zur Berechnung der Integrale $C_{\hat{\lambda}}^{\mu_{1} \ldots \mu_{n}}$ und $D$ sind im wesentlichen von H. Goenner entwickelt worden. Wir danken Herrn Dr. Goenner herzlich für diese und andere wertvolle Beiträge zu unserem Thema (vgl. auch [35]).

\section{Literatur}

1. Infeld,L., Plebański, J.: Motion and relativity. London: Pergamon Press 1960.

2. Burke, W.L.: J. Math. Phys. 12, 401 (1971).

3. Lubanski, J.: Acta Phys. Polon. 6, 356 (1937).

4. Sachs, R., Bergmann, P.G̈.: Phys. Rev. 112, 674 (1958).

5. Westpfahl, K.: Ann. Physik 20, 112 (1967).

6. Bertotti, B.: Nuovo Cimento 4, 898 (1956).

7. Havas, P.: Phys. Rev. 108, 1351 (1957).

8. Geissler, D.: Z. Naturforsch. 14a, 689 (1959).

9. Kerr, R.: Nuovo Cimento 13, 469, 492, 693 (1959).

10. Havas, P., Goldberg, J.: Phys. Rev. 128, 398 (1962).

11. Smith, S., Havas, P.: Phys. Rev. 138, B 495 (1965).

12. Goenner, H., Gralewski, U., Westpfahl, K.: Z. Physik 207, 186 (1967).

13. Bertotti, B., Plebański, J.: Ann. Phys. (N. Y.) 11, 169 (1960).

14. Stephani, H.: Acta Phys. Polon. 26, 1045 (1964).

15. Kühnel, E.: Ann. Phys. (N. Y.) 28, 116 (1964).

16. Newman, E., Penrose, R.: J. Math. Phys. 3, 566 (1962).

17. Bondi, H., Burg, M. van der, Metzner, A.: Proc. Roy. Soc. 269, 21 (1962).

18. Sachs, R.: Proc. Roy. Soc. 270, 103 (1962).

19. Janis, A., Newman, E.: J. Math. Phys. 6, 902 (1965).

20. Bonnor, W., Rotenberg, M.: Proc. Roy. Soc. 289, 247 (1966).

21. Tamburino, L., Winicour, J.: Phys. Rev. 150, 1039 (1966).

22. Torrence, R., Janis, A.: J. Math. Phys. 8, 1355 (1967). 
23. Couch, W., Torrence, R., Janis, A., Newman, E.: J. Math. Phys. 9, 484 (1968).

24. Hunter, A., Rotenberg, M.: J. Phys. A (Gen. Phys.) 2, 34 (1969).

25. Hallidy, W., Janis, A.: J. Math. Phys. 11, 578 (1970).

26. Couch, E., Torrence, R.: J. Math. Phys. 11, 2096 (1970).

27. Dirac,P.A. M.: Proc. Roy. Soc. 167, 148 (1938).

28. Haag, R.: Z. Naturforsch. 10a, 752 (1955).

29. Westpfahl, K.: Fortschr. Phys. 15, 309 (1967).

30. Römer, H., Westpfahl, K.: Ann. Physik 22, 264 (1969).

31. Bennewitz,F.: Dissertation, Freiburg/Brsg. 1967 (unveröff.).

32. Fock, V.A.: Theorie von Raum, Zeit und Gravitation. Berlin: Akademie-Verlag 1960,

33. Van Dam, H., Wigner, E. P.: Phys. Rev. 138, B 1576 (1965).

34. - - Phys. Rev. 142, 838 (1966).

35. Goenner,H.: Dissertation, Freiburg/Brsg. 1966 (unveröff.).

Prof. Dr. K. Westpfahl

Dr. F. Bennewitz

D-7800 Freiburg i. Br.

Hermann-Herder-Str. 3

Deutschland 